\title{
Im Zerrspiegel des Populismus
}

\section{Eine computergestützte Analyse der Verlinkungspraxis von Bundestagsabgeordneten auf Twitter}

\author{
Gerret von Nordheim • Jonas Rieger
}

Online publiziert: 29. Juni 2020

(C) Der/die Autor(en) 2020

Zusammenfassung Die vorliegende Studie betrachtet die Praxis des Teilens von Texten durch politische Akteure auf Twitter und analysiert den thematischen Geltungsanspruch, der sich darin spiegelt: zugespitzt populistisch oder vielfältig und inklusiv im Stil einer Volkspartei. Konkret wurde das ,Verlinkungsverhalten' deutscher Bundestagsabgeordneter auf Twitter untersucht - mit besonderem Augenmerk auf der rechtspopulistischen AfD. Hierzu wurden mit Hilfe eines adaptiven Scrapers 56.107 Texte gesammelt, die Bundestagsabgeordnete in der laufenden Legislaturperiode in Tweets geteilt hatten. Der Analyse ihrer Vielfalt diente eine Maßzahl, die thematische Heterogenität auf Basis von Topic-Modellen ermittelt. Die Resultate zeigen, dass populistische Akteure zwar häufiger als andere auf Medien verlinken und dabei heterogene Quellen nutzen, dass sich dies jedoch nicht in thematische Vielfalt übersetzt, im Gegenteil: Die Themenagenda der AfD-Parlamentarier ist weniger vielfältig als die der Politiker anderer Parteien. Die klar populistische Themenselektion korreliert zudem stärker mit metrischem Erfolg (in Form von Likes, Retweets), als dies bei den Themen anderer Parteien der Fall ist.

Schlüsselwörter Populismus · Gatekeeping · Vielfalt · Twitter · Topic Modeling

Die Studie ist ausschließlich mit Mitteln der unten genannten Hochschulen finanziert worden und ist nicht im Rahmen einer Lehrveranstaltung entstanden.

Dr. G. von Nordheim $(\bowtie)$

Sozialwissenschaften, Journalistik und Kommunikationswissenschaft, Universität Hamburg, 20146 Hamburg, Deutschland

E-Mail: gerret.vonnordheim@uni-hamburg.de

\section{J. Rieger}

Fakultät für Statistik, Technische Universität Dortmund, 44221 Dortmund, Deutschland

E-Mail: rieger@statistik.tu-dortmund.de 


\section{Distorted by populism}

A computational analysis of German parliamentarians' linking practices on Twitter

Abstract Hybrid media systems offer an opportunity for political actors to bypass traditional gatekeepers and communicate directly with their electorate. While in the past, politicians of traditional parties have been rather hesitant to adopt this option, representatives from the new populist parties, in particular, are using the direct channel of social media intensively and successfully. What is more, it seems that the platforms' communication conditions are virtually perfect for making populist appeals.

In this context, sharing journalistic contents is of major importance. Referencing traditional media underscores a party's own claim to be a legitimate voice in the political discourse. At the same time, populists use the re-contextualization logic that is inherent in the hyperlink structure in order to generate topic saliencies that are in line with their ideological agenda. So far, the implications of this shift in the gatekeeper instance on contents remain unclear. Studies at the source level, such as numerical counts of linked media brands (source diversity), fall short of describing this phenomenon and underestimate fragmentation effects because they do not offer any insights how curating specific contents can have a polarizing effect on user's or user group's construction of reality.

The question how "secondary gatekeeping" on digital platforms can lead, for instance, to a concentration of specific topics (i.e., a loss of diversity) along specific group identities will require an analysis of the contents that are associated with such links.

To do this, this study uses an adaptive scraper that extracts text data in a structured form independent of the structure of the web page that contains them. We were thus able to save 56,107 associated text files from 846,744 tweets (with 151,885 links) posted by members of the German Bundestag on Twitter. Based on this generated corpus, we used topic clustering to analyze the thematic structure of the shared content and determine its relative diversity.

The central results of the study are:

1. The topic agenda of populist actors (our example being the AfD) differs starkly from the other parties' topic priorities. Its structure is different-heavily addressing a small number of topics - and it is focused on different types of contents (see below). Compared to the other parties' sharing behavior on Twitter, the AfD's links reflect a much less diverse topic agenda.

This confirms findings from previous studies that right-wing populists' sharing practices reinforce the identity of their primary target group. It seems that sharing journalistic contents is a central part of their populist communication strategy: While AfD politicians are otherwise no more active on Twitter than other parliamentarians, they clearly share a lot more.

2. Topic selection by populist actors correlates more strongly with the metric success of individual topics than is the case with other parties-clearly demonstrating a synergetic relationship.

The topics that literature describes as characteristic of populists are mirrored in the topics with which AfD politicians trigger the most engagement: migration, 
the negotiation of identity in relation to right-wing extremism, and minorities (i.e. outgroups), crime, i.e. corruption and other crimes, as well as the discussion of economic hardship, especially on issues of opportunity and distribution.

The methodological perspective confirms that an analysis of external diversity leads to different conclusions than an analysis of internal diversity. If one were to compare the diversity of linked media alone, one might infer that the AfD's gatekeeping does not differ significantly from that of the other parties. A mere focus on external diversity could thus contribute to a normalization of populist communication. This demonstrates the added value of content-level analysis (rather than just at source level). Contrasting the two levels also allows us to apply the "populist communication paradox" to gatekeeping: Linking traditional journalistic sources lends credence to the underlying populist agenda-and paradoxically, this also acts as a distorting mirror of the journalistic topic agenda that supports the populist criticism of journalistic agenda-setting.

The present study also offers interesting insights into the other parties: The coalition parties, for instance, are strikingly similar across all measured dimensions. This confirms earlier studies on the proximity of the governing parties in terms of content. In line with earlier studies, we can confirm that the Green Party's social media behavior, in particular, differs from that of the other parties. In several respects, they represent the counter-model to AfD: They have the most diverse topic agenda and their selection of topics shows the least correlation with engagement metrics. While AfD stands out for its issue-ownership of typically populist topics and the associated loss of diversity, the Green Party communicates broadly and on a variety of topics.

Keywords Populism · Gatekeeping · Diversity · Twitter · Topic Modeling

\section{Einleitung}

Politische Akteure haben in hybriden Mediensystemen die Möglichkeit, klassische Gatekeeper zu umgehen und direkt mit der Wählerschaft zu kommunizieren. Während Politiker klassischer Parteien diese Möglichkeit in der Vergangenheit eher zögerlich ergriffen (für Deutschland vgl. Oelsner und Heimrich 2015, S. 466), zeichnen sich Repräsentanten neuer populistischer Parteien insbesondere dadurch aus, dass sie die direkte Verbindung durch soziale Medien intensiv und erfolgreich nutzen (vgl. Engesser et al. 2017; Ernst et al. 2018). Mehr noch: Es scheint, als seien die Kommunikationsbedingungen, die die Plattformen bieten, wie gemacht für die populistische Ansprache (vgl. Gerbaudo 2018; Dittrich 2017).

Der Praxis des Teilens journalistischer Inhalte kommt hierbei eine besondere Bedeutung zu. Der Verweis auf etablierte Medien unterstreicht den eigenen Geltungsanspruch als legitime Partei im politischen Diskurs. Gleichzeitig nutzen Populisten die der Hyperlink-Struktur innewohnende Rekontextualisierungslogik, um entsprechend ihrer ideologischen Ausrichtung Themen besonders hervorzuheben. Es ist bisher unklar, wie sich diese Verschiebung der Gatekeeper-Instanz inhaltlich auswirkt. Untersuchungen auf Quellenebene, beispielsweise die Zählung verlinkter Medienmarken, werden dem Phänomen nicht gerecht. Sie unterschätzen zudem 
mögliche Fragmentierungseffekte (vgl. Bechmann und Nielbo 2018, S. 999), da sie keine Aussagen darüber erlauben, ob verschiedenen Usergruppen durch unterschiedlich präsentierte Inhalte unterschiedliche Bilder von Realität vermittelt werden. Wie Stier et al. konstatierten, betrachtet beispielsweise nur ein kleiner Teil der Untersuchungen politischer Social-Media-Nutzung ,the actual contents of communication going beyond meta data“ (Stier et al. 2018, S. 52). Die Frage, inwiefern secondary gatekeeping (vgl. Singer 2013) auf digitalen Plattformen beispielsweise zur Konzentration bestimmter Themen (also zu Vielfaltsreduktion) entlang spezifischer Gruppenidentitäten führt, lässt sich nur durch eine Analyse der mit den Links assoziierten Inhalte beantworten.

Um dieses Ziel zu erreichen, haben wir für diese Studie einen adaptiven Scraper eingesetzt, der unabhängig vom Aufbau einer Webseite enthaltene Textdaten in strukturierter Form extrahiert. So war es uns möglich, aus 846.744 von Bundestagsabgeordneten geposteten Tweets 56.107 assoziierte Textdateien zu speichern. Mit diesem Korpus haben wir durch Topic-Clustering die thematische Struktur der geteilten Inhalte analysiert und ihre relative Vielfalt bestimmt. Damit war es möglich, Akteure zu identifizieren, die als Gatekeeper vielfältige Gesellschaftsbereiche ansprechen, sowie solche, die der Öffentlichkeit einen Zerrspiegel vorhalten, indem sie primär Themen bedienen, die ihre Follower in ihrem Weltbild bestärken. Dass diese Strategie im Sinne der Engagement-Logik der Plattformen rational ist, zeigt die hohe Korrelation von populistischer Themenselektion und metrischem Erfolg.

\section{Theoretischer Rahmen und Forschungsstand}

\subsection{Populistisches Gatekeeping und Identität}

Populismus lässt sich als Organisationsform (ausgerichtet auf einen charismatischen Führer), als Kommunikationsstil (ohne intermediäre Instanzen, direkt zum Volk) oder beispielsweise als Diskurs (mit bestimmten rhetorischen Mustern) beschreiben. Wie Mudde jedoch betont, katalysieren diese Ausprägungen Populismus eher, als dass sie ihn definierten. Er selbst bezeichnet Populismus daher als dünne Ideologie (vgl. auch Freeden 2008, S. 485),

„that considers society to be ultimately separated into two homogeneous and antagonistic groups, , the pure people 'versus, the corrupt elite', and which argues that politics should be an expression of the volonté générale (general will) of the people. " (Mudde 2004, S. 543, Hervorhebung im Original)

Die programmatische Leere der dünnen Ideologie sei es, die sie für verschiedene andere Ideologien anschlussfähig mache, beispielsweise Kommunismus, Ökologismus, Nationalismus oder Sozialismus (vgl. Mudde 2004, S. 544). Populismus manifestiert sich dementsprechend medial in einer beschränkten Anzahl spezifischer Themen. Ernst et al. (vgl. 2018, S. 172) sprechen von fünf populistischen Kernthemen: Migration, (regionale) Identität, Korruption und Verbrechen, (europäische/transnationale) Integration und wirtschaftliche Not. Dieses Agenda Setting wird mit verschiedenen kommunikativen Strategien aus der Berichterstattung derjenigen 
journalistischen Medien abgeleitet, zu denen sich populistische Akteure ansonsten antagonistisch positionieren - Haller und Holt sprechen hierbei von ,paradoxical populism“ (vgl. Haller und Holt 2019).

Dieses Phänomen lässt sich insbesondere in den Kanälen sozialer Medien beobachten. Sie bilden die idealen „opportunity structures“ (Ernst et al. 2018) für populistische Kommunikation. Hier sind es nämlich nicht mehr traditionelle Massenmedien, die Aufmerksamkeit lenken - Thematisierungsprozesse werden vielmehr durch sozio-technologische Prozesse gesteuert und lassen sich als sekundäres (vgl. Singer 2013, S. 56) und kollektives Gatekeeping (vgl. Keyling 2017, S. 78-105) beschreiben.

So ist das Teilen massenmedialer Inhalte ein effektives Werkzeug, um sich einerseits als Teil des „Mainstream“-Diskurses zu legitimieren und sich andererseits abzugrenzen. Die Wahl (also auch die Empfehlung) von Medieninhalten ist in diesem Sinne nicht nur eine Frage der Informationsbeschaffung - sie ist vielmehr Vehikel von Selbstwahrnehmung und sozialer Identität (vgl. Ihm und Kim 2018, S. 4360; Slater 2007, S. 291) und wird als solches von strategisch-professionell agierenden Gatekeepern eingesetzt (,strategic professionals“, Wallace 2018, S. 280). Diese Strategie ist besonders vielversprechend bei Individuen, die extreme rechtspopulistische Ideologien vertreten; homogene, bestätigende Medien wirken in diesem Umfeld besonders identitätsstiftend (vgl. Dvir-Gvirsman 2017, S. 1086).

In der Folge führt dies zu stark divergierenden issue agendas auf politischer Ebene, die auf soziale und affektive Polarisierung hindeuten (vgl. Suhay et al. 2017, S. 97). Gleichzeitig zeigen ältere theoretische Konzepte, beispielsweise die Idee der issue ownership (vgl. Petrocik 1996), dass es zu einem klassischen Merkmal ausdifferenzierter Parteiensysteme gehört, Themen parteipolitisch zu besetzen.

\subsection{Populismus und soziale Medien}

Die populistische Logik scheint mit Medien in einem synergetischen Funktionsverhältnis zu stehen: Gerbaudo weist in diesem Sinne auf eine „elective affinity“ (vgl. Gerbaudo 2018, S. 746) hin. Erfolg in den sozialen Medien sei ein hervorstechendes Merkmal verschiedener populistischer Parteien und Bewegungen. Die ,condition of atomization“ innerhalb der sozialen Medien sei ,ultimately conducive to the populist logic which is centrally concerned with fusing atomized individuals in the collective body of the people“ (Gerbaudo 2018, S. 748). Das sekundäre Gatekeeping ist nur als Resultat dieses Zusammenwirkens sozialer und technologischer Prozesse zu verstehen - Atomisierungs- und Kristallisationsprozesse sind der populistischen wie der algorithmischen Logik eigen (vgl. Krämer 2017, S. 1305). Dittrich veranschaulicht am Beispiel von Facebook, wie sich diese Symbiose in den Relevanzoder Aufmerksamkeitsindikatoren der sozialen Medien spiegelt - populistische Akteure lösen deutlich mehr Interaktionen und Engagement aus als die Seiten anderer Parteien (vgl. Dittrich 2017, S. 11). 


\subsection{Externe und interne Vielfalt}

Populisten konzentrieren sich wie beschrieben vor allem auf bestimmte Kernthemen - es ist daher zu erwarten, dass mit dem populistischen Gatekeeping eine Reduktion von Themenvielfalt einhergeht. Unklar ist hingegen, ob dies auch zu einer Beschränkung auf wenige Medienmarken führt. Im Gegenteil erscheint es bei einer Spezialisierung auf einen kleinen Ausschnitt der allgemeinen Medienagenda rational, möglichst viele Quellen einzubeziehen. Darüber hinaus entspräche es dem Kalkül der beschriebenen Legitimationsstrategie, die Anzahl „opportuner Zeugen“ (Hagen 1992, S. 449) zu maximieren, um die Relevanz populistischer Themen für die Gesellschaft zu unterstreichen.

Es erscheint daher von äußerster Wichtigkeit, terminologisch insbesondere zwischen der Vielfalt verlinkter Medien und der Vielfalt der verlinkten Inhalte zu unterscheiden. Schon zu Zeiten, in denen programmatisch-gebündelte Angebote den Medienmarkt dominierten, wurde darauf hingewiesen, dass die bloße Anzahl medialer Anbieter nicht mit der inhaltlichen Vielfalt ihres Angebots gleichgesetzt werden sollte (vgl. Van Cuilenberg 1999, S. 189). Vor dem Hintergrund heutiger Möglichkeiten der Individualisierung von Medienkonsum scheint es umso eher geboten, beides getrennt zu betrachten.

Terminologisch anschlussfähig erscheint hier die Trennung zwischen externer und interner Vielfalt (vgl. McQuail und Van Cuilenburg 1983, S. 151). Interne Vielfalt beschreibt die Inhaltsvielfalt (content diversity, vgl. Napoli 1999, S. 18-24), die sich als Pluralität von Akteuren, Sichtweisen oder Themen (siehe auch ,,media agenda diversity “, Tan und Weaver 2013, S. 774) manifestieren kann - ursprünglich innerhalb eines Mediums. Externe Vielfalt beschreibt das Vorhandensein verschiedener Medien und ihre Varianz hinsichtlich bestimmter Charakteristika ${ }^{1}$ - ursprünglich vor allem innerhalb eines Medienmarktes. Beide Termini lassen sich für die Analyse sekundärer Gatekeeping-Prozesse adaptieren.

\subsection{Thematische Expansion}

Während das populistische Gatekeeping wie oben beschrieben einer thematischen Engführung gleichkommt, kann es aus der Perspektive politischer Kommunikation ebenso rational sein, eine breite Themenpalette zu bedienen, um eine maximal große Wählerschaft anzusprechen. Diesem Kalkül folgend, ist es ferner plausibel anzunehmen, dass der Populismus in seiner parteipolitischen Ausprägung einen Ausgleich beider Strategien anzustreben versucht: identitätsstiftende Konzentration auf seine Kernthemen, gleichzeitig Kommunikation eines universellen Anspruchs, der politische Eignung (bzw. Regierungsfähigkeit) unterstreicht. Krämer beschreibt in diesem Sinne, wie sich rechter Populismus online sukzessiv von einer thin ideology zu einer full-blown ideology entwickele: ,[W]e may witness the development of distinctive-

\footnotetext{
${ }^{1}$ Oftmals auch als Quellenvielfalt (source diversity, vgl. Napoli 1999, S. 11-18) bezeichnet - der Gebrauch des Begriffs ist jedoch nicht einheitlich, er wird auch als Dimension inhaltlicher Vielfalt genutzt (als Vielfalt der journalistisch verwendeten Quellen, vgl. Voakes et al. 1996, S. 583-584).
} 
ly right-wing populist elements in the lifestyle of distinct milieus." (Krämer 2017, S. 1297).

Am Ende dieses Abschnitts steht also die Frage, welche theoretische Perspektive den Status Quo beschreibt: Sind soziale Medien in der Hand der Populisten Werkzeuge, die ihnen ermöglichen, mit Hilfe entbündelter Inhalte auf ihre Kernthemen aufmerksam zu machen? Oder lassen sich Indizien dafür finden, dass die thematischen Geltungsansprüche populistischer Kommunikation mittlerweile umfassender sind? Durch die Wahlerfolge rechtspopulistischer Parteien und die Besetzung politischer Positionen stehen der institutionalisierten populistischen Kommunikation heute deutlich mehr Ressourcen zur Verfügung als noch vor einigen Jahren. Es ist daher von äußerster Relevanz zu klären, inwiefern sich populistische Kommunikation professionalisiert hat und ob sich der neue umfassende Geltungsanspruch ${ }^{2}$ in ihrer medialen Praxis spiegelt.

Auf diese Fragen suchen wir Antworten, indem wir das Kommunikationsverhalten populistischer Akteure relativ zum Verhalten anderer politischer Akteure analysieren. Dazu betrachten wir insbesondere die Praxis des Verlinkens als Mittel des sekundären Gatekeepings. Sie stellt einen geeigneten Messpunkt dar, da sich hier der thematische Geltungsanspruch einer Partei spiegelt. Konkret soll das ,Verlinkungsverhalten' deutscher Bundestagsabgeordneter auf Twitter untersucht werden.

In einer der wenigen parteivergleichenden Inhaltsanalysen aus Deutschland fanden Caton et al. (2015, S. 8) im Kontext der Bundestagswahl 2013, dass sich insbesondere die Facebook-Posts größerer Parteien (CDU/CSU und SPD) ähneln ähnlich folgert Tromble: „For parties in government, cohesion helps retain power.“ (Tromble 2016, S. 679) Auf der anderen Seite zeichnen sich kleinere Parteien jeweils durch eigene Charakteristika aus. Insbesondere Die Grünen stachen in der Untersuchung von Caton et al. als ,least similar and most future-oriented party“ (Caton et al. 2015, S. 14) hervor. Es gibt bisher keine Erkenntnisse dazu, wie sich die AfD relativ zu den anderen Parteien in diesem Koordinatensystem positioniert.

\section{Hypothesen}

Vor dem Hintergrund der dargestellten Forschung erscheint es plausibel anzunehmen, dass die Praxis des Verlinkens besonders intensiv von rechtspopulistischen Akteuren als Mittel des sekundären Gatekeepings genutzt wird - wiewohl populistische Kommunikationsweisen im gesamten politischen Spektrum zu beobachten sind. Besonderes Augenmerk liegt in dieser Untersuchung daher auf der AfD als einziger rechtspopulistischer Partei im Bundestag (vgl. Rooduijn et al. 2019). Gleichzeitig soll das Kommunikationsverhalten der anderen Parteien im Verhältnis zur AfD betrachtet werden.

Die dargestellten Evidenzen deuten außerdem mehrheitlich darauf hin, dass es insbesondere beim populistischen Gatekeeping zu einer inhaltlich-thematischen Reduktion der Agenda auf typische Kernthemen kommt und dass dieses Verhalten in

\footnotetext{
2 Vgl. bspw. AfD-Bundessprecher Jörg Meuthen nach den Landtagswahlen in Sachsen und Brandenburg: „Wir haben uns als Volkspartei etabliert.“ (zit. n. May 2019)
} 
einem synergetischen Verhältnis zu den Engagement-Metriken der sozialen Medien steht.

Aus diesen Punkten ergeben sich für diese Arbeit folgende Hypothesen:

H1 Im Vergleich zur Verlinkungspraxis der anderen Parteien auf Twitter spiegelt sich in den von der AfD verlinkten Texten eine weniger vielfältige Themen-Agenda.

H2 Themen, die von der AfD besetzt werden, sind - gemessen an EngagementMetriken (Likes, Shares) - erfolgreicher als Themen anderer Parteien auf Twitter.

\subsection{Daten: Beschaffung}

Den Ausgangspunkt der Untersuchung bilden 846.744 von Bundestagsabgeordneten $^{3}$ gepostete Tweets, die via API (und rtweet, vgl. Kearney 2019) gesammelt und jeweils einer Partei zugeordnet wurden. Die Tweets wurden im Oktober 2019 gespeichert - redundante und alle vor dem 24.10.2017 veröffentlichten Tweets wurden gelöscht, so dass der Datensatz ausschließlich singuläre und in dieser Legislaturperiode veröffentlichte Posts enthält (übrig blieben 515.782 Tweets) ${ }^{4}$. Insgesamt wurden in $29 \%$ dieser Tweets Links gepostet (151.885 Links zu insgesamt 132.582 verschiedenen URLs).

Es wäre mit herkömmlichen Scrapern, die an jede Seitenstruktur angepasst werden müssen und nur bedingt generalisierbar sind, forschungsökonomisch nicht realisierbar gewesen, diesen heterogenen URL-Datensatz auszulesen. Um dennoch ein Korpus generieren zu können, haben wir den kostenpflichtigen Service Diffbot genutzt $^{5}$. Der Anspruch des kalifornischen Unternehmens ist es, ,to create an autonomous system that can read and understand all of the documents on the public web, convert these to structured entities, and make this accessible as a service to others“" (Diffbot 2019). Mit Hilfe des von Diffbot bereitgestellten R-Clients (vgl. Tung 2019) wurden im nächsten Schritt die mit den (zuvor formatierten und bereinigten ${ }^{6}$ ) Links assoziierten Texte bezogen. Die API des Service gibt eine strukturierte Textdatei aus, in der neben dem Artikel beispielsweise Überschrift, Datum, Autor erkannt werden. Insgesamt konnten so für $72 \%$ der über Diffbot abgefragten Links Texte heruntergeladen werden $(N=60.667)$.

\footnotetext{
3 Eine aktuelle Twitter-Liste aktiver Bundestagspolitiker führt der Blogger Martin Fuchs (2019).

${ }^{4}$ Bei insgesamt 42 Politikern konnten nicht alle während dieser Legislaturperiode veröffentlichten Tweets aufgenommen werden, da sie in dieser Zeit mehr als 3200 Tweets gepostet haben (die Twitter-API erlaubt, maximal 3200 Tweets pro Account zu speichern).

5 Wir würden aus forschungsethischen Gründen die Verwendung einer Opensource-Software vorziehen. Die vorliegende Studie wäre mit den uns bekannten Opensource-Alternativen allerdings nicht forschungsökonomisch durchführbar. Wir halten die Verwendung des kommerziellen Dienstes daher für legitim. Darüber hinaus sind drei Gründe zu nennen, die unseres Erachtens die Verwendung von Diffbot rechtfertigen: 1. Es werden keine sensiblen oder rechtlich geschützten Daten übertragen, 2. der Preis ist verglichen mit anderen Software-Lizenzen niedrig und erzeugt damit geringe Hürden bei der Replizierbarkeit etc., 3. die Ergebnisse des Dienstes lassen sich überprüfen.

${ }^{6}$ Es wurden Links auf Twitter, Instagram, Youtube und automatisch generierte Performance-Tweets (z. B. „Sie haben seit gestern X neue Follower“") entfernt, da hier keine verwertbaren Texte zu erwarten waren.
} 
Bei den nicht erfolgreich gespeicherten Webseiten handelte es sich zum Großteil um Bilddaten oder Multimedia-Websites (Videos, interaktive Grafiken etc.). Der Scraper versagte nur bei wenigen Webseiten systematisch. Von den für die Untersuchung besonders relevanten Seiten waren es einzig Inhalte der Domain Bild.de, die Diffbot nicht erkennen konnte und die mit Hilfe eines zweiten, browserbasierten Scrapers (vgl. Octoparse 2019) ergänzt werden mussten. Der so zusammengetragene Datensatz war weiter zu bereinigen?: Texte mit weniger als 15 Wörtern wurden entfernt. Außerdem wurden längere Texte randomisiert gekürzt (auf das dritte Quartil der Textlängen $\hat{=} 396$ Wörter), um einen übermäßigen Einfluss langer Texte auf das Themenmodell zu vermeiden. In die Vokabelmenge wurden 76.527 unterschiedliche Worte (types) aufgenommen (Kriterien: zwischen 3 bis 39 Zeichen und mehr als zehn Entsprechungen im reduzierten Datensatz).

Texte, die keine (oder nicht hauptsächlich) Wörter der deutschen Sprache enthielten, wurden eliminiert (beispielsweise fremdsprachige Texte oder Blindtexte, die beim Scrapen von verschlüsselten Bezahlartikeln gespeichert werden). Hierzu wurde mit Hilfe des R-Pakets spelling (vgl. Ooms und Hester 2019) der Anteil deutscher Wörter in den Texten identifiziert - Texte, deren Wörter zu 30 oder mehr Prozent ${ }^{8}$ aus nicht-deutschen Vokabeln bestanden, wurden gelöscht. Diese Vorverarbeitung resultierte in einem Korpus mit insgesamt 56.107 Artikeln.

\subsection{Daten: Beschreibung}

\subsubsection{Politiker/Parteien auf Twitter}

Im Oktober 2019 waren 536 Bundestagsabgeordnete auf Twitter vertreten - das entspricht $76 \%$ aller Parlamentarier (522 davon im gesamten Datensatz enthalten, 479 im reduzierten/gesäuberten). Ein Vergleich der Ergebnisse mit früheren Studien zeigt deutlich, dass die Twitter-Adoption der Politiker in den vergangenen Jahren in Wellen verlief: Ende 2010 waren $33 \%$ aller Bundestagsabgeordneten aktiv auf Twitter (vgl. Saalfeld und Dobmeier 2012, S. 320). Dieses Niveau stagnierte zunächst, auch 2012 hatten nicht mehr als ein Drittel der Parlamentarier einen Account (vgl. Oelsner und Heimrich 2015, S. 459). Ein Jahr später jedoch, zur Bundestagswahl 2013, waren bereits mehr als die Hälfte der Politiker auf Twitter aktiv (vgl. Nuernbergk und Conrad 2016, S. 4), vier Jahre später zur nächsten Wahl 65 \% (vgl. Schmidt 2017, S. 616). Die Aktivierung der Accounts ist eindeutig assoziiert mit den Bundestagswahlen. Dies bestätigt auch eine Analyse der Entstehungszeitpunkte der Accounts (siehe Abb. 1). In der laufenden Legislaturperiode sind 12\% (63 Accounts) der heute aktiven Twitter-Accounts hinzugekommen. Am aktuellen Rand sind es besonders Politiker von AfD (gegründet 2013) und FDP (in der letzten Legislaturperiode nicht im Bundestag vertreten), die neue Twitter-Accounts eingerichtet haben.

\footnotetext{
7 Die komplette Liste der Vorverarbeitungsschritte und der zugehörige R-Code können bei den Autoren angefragt werden.

8 Die Grenze wurde auf Basis der Codierungen eines Samples von 450 Texten validiert.
} 


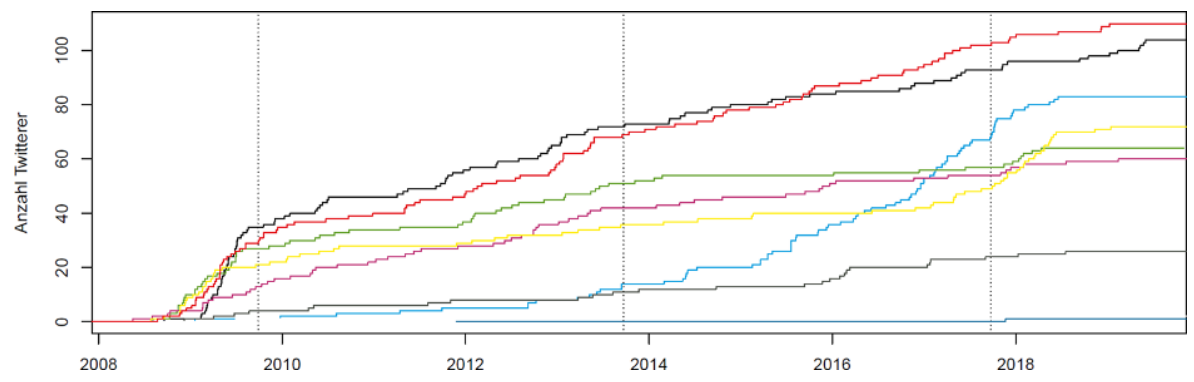

Abb. 1 Die Kurven beschreiben die aggregierten Zahlen von Twitter-Accounts der Bundestagsabgeordneten jeder Partei. Die verschiedenen Fraktionen sind in ihren geläufigen Farben dargestellt (Die Linke in Purpur, SPD in Rot, Die Grünen in Grün, CDU/CSU in Schwarz, FDP in Gelb und AfD in Blau). Die vertikalen Linien markieren Bundestagswahlen

Analysiert man die Parteienzusammensetzung, ergibt sich folgendes Bild ${ }^{9}: 25 \%$ aller Parlamentarier auf Twitter gehören CDU oder CSU an, 22\% der SPD, 11\% der Linken, $12 \%$ den Grünen, $16 \%$ der AfD, $14 \%$ der FDP. Gemessen an der Zahl der Accounts sind Politiker der Partei Die Grünen die aktivsten Twitterer - 96\% aller Grünen-Politiker im Bundestag sind auf Twitter vertreten (92\% AfD, $91 \%$ FDP, $87 \%$ Die Linke, $76 \%$ SPD, $55 \%$ CDU/CSU). Gemessen an der Anzahl der Posts im Datensatz sind SPD-Politiker die aktivsten - 22\% aller Tweets stammen von SPDPolitikern (CDU/CSU 20\%, Die Grünen 18\%, Die Linke 16\%, FDP 12\%, AfD $10 \%)$. Gemessen an den Verlinkungen in den Posts ergibt sich ein leicht verändertes Bild: Hier ist die AfD die aktivste Partei - 23\% aller Link-Posts im gesäuberten Datensatz stammen von Abgeordneten der rechtspopulistischen Partei (20\% Die Grünen, $18 \%$ Die Linke, $13 \%$ FDP, $12 \%$ CDU/CSU, $12 \%$ SPD).

\subsubsection{Verlinkte Webseiten/Medien}

Betrachtet man die verlinkten Webseiten und Medien, wird deutlich, dass journalistische Nachrichtenmedien mit deutlichem Abstand zu den meistverlinkten Seiten gehören: Im analysierten URL-Datensatz befinden sich unter den 62 Seiten, die mehr als 100-mal geteilt wurden, nur zehn Seiten ohne redaktionelles Angebot ${ }^{10}$ : die Webseiten der Fraktionen (AfD, CDU/CSU, SPD, FDP, Grüne), die Webpräsenzen des Auswärtigen Amts und des Bundestags, zwei Seiten einzelner Politiker sowie die Plattform Facebook.

Vergleicht man die von den Parteien verlinkten Medien, ist es schwierig, gravierende Unterschiede festzustellen. Selbst die von der AfD am häufigsten verlinkten Seiten setzen sich fast ausschließlich aus journalistischen Medien zusammen (siehe Tab. 1). Die AfD-Politiker teilen sowohl Inhalte von Medien, die als konservativ

\footnotetext{
9 Parlamentarier der Partei „Die Blauen“ $(N=2)$ sowie parteilose Bundestagsabgeordnete $(N=2)$, d.h. fraktionslose Abgeordnete, werden hier nicht gesondert aufgeführt.

${ }^{10}$ Subsumiert wurden unter diese Kategorie neben klassischen journalistischen Medienmarken auch populistische Publikationen (Tichys Einblick, Junge Freiheit), satirische Seiten (Der Postillon) und Blogs wie Netzpolitik.
} 
Tab. 1 Die 15 Webseiten, die am häufigsten von Parlamentariern der AfD verlinkt wurden (Grundmenge: gesäuberter URL-Datensatz); zusätzlich die Anzahl der Posts pro Partei, die auf Seiten der Domain verlinken

\begin{tabular}{lllllll}
\hline & AfD & Die Grünen & Die Linke & FDP & SPD & CDU/CSU \\
\hline Welt & 2418 & 523 & 336 & 940 & 264 & 645 \\
Bild & 957 & 58 & 32 & 261 & 80 & 283 \\
Focus & 955 & 72 & 128 & 218 & 67 & 156 \\
Junge Freiheit & 926 & 0 & 0 & 0 & 0 & 0 \\
FAZ & 605 & 355 & 208 & 581 & 145 & 365 \\
Spiegel & 574 & 1346 & 1235 & 700 & 1362 & 423 \\
Tichys Einblick & 341 & 0 & 0 & 49 & 4 & 10 \\
n-tv & 303 & 102 & 142 & 205 & 54 & 150 \\
Tagesspiegel & 287 & 547 & 359 & 373 & 262 & 189 \\
AfD-Bundestag & 209 & 0 & 0 & 0 & 0 & 0 \\
Zeit & 205 & 368 & 291 & 180 & 193 & 83 \\
Süddeutsche & 185 & 817 & 454 & 245 & 508 & 156 \\
Tagesschau & 185 & 478 & 395 & 250 & 187 & 454 \\
BZ-berlin & 144 & 30 & 15 & 31 & 13 & 55 \\
NZZ & 144 & 31 & 24 & 71 & 13 & 36 \\
\hline
\end{tabular}

gelten, als auch Links zu liberalen Medien, Boulevard-Inhalte genauso wie Texte aus Qualitätszeitungen.

Vergleicht man nun die externe Vielfalt anhand der Anzahl der unterschiedlichen verlinkten Informationsmedien ${ }^{11}$ pro Partei, ergibt sich folgendes Bild: Grünen-Politiker verlinkten auf 179 verschiedene Quellen, die AfD auf 175, die Linke auf 174, FDP auf 165, SPD auf 164, CDU/CSU auf 157.

\section{Methode}

Topic-Modeling-Verfahren wie LDA (vgl. Blei et al. 2003) ermöglichen es, latente thematische Zusammenhänge in großen Textkorpora sichtbar zu machen. Der Ausgangspunkt dieser automatischen Mustererkennung ist die Korrelation zwischen der Bedeutungsähnlichkeit linguistischer Einheiten (Wörter, Phrasen) und der Ähnlichkeit ihrer Verteilung über bestimmte sprachliche Kontexte. Bei der LDA werden diese Zusammenhänge als Topics modelliert, als Wahrscheinlichkeitsverteilungen über alle Wörter. Jedes Dokument im Korpus kann ebenfalls als Wahrscheinlichkeitsverteilung (über die Topics) dargestellt werden. Die Topics der LDA können grobe Themenstrukturen aber auch feinere Zusammenhänge spiegeln - ihre Granularität ist abhängig von verschiedenen Variablen: der Beschaffenheit des Korpus, der Länge und der Anzahl der Dokumente, ihrer thematischen Durchmischung und

\footnotetext{
11 In dieser Auswertung wurden alle Quellen einbezogen, die mindestens zehnmal verlinkt wurden (296 Seiten, assoziiert mit 88,5\% aller Verlinkungen) - abgezogen wurden politische und staatliche Informationsangebote, also beispielsweise Politikerblogs, Parteiseiten, Seiten von Ministerien etc. (resultierend in einer Liste von 206 Seiten).
} 
Zusammensetzung; aber auch von den Voreinstellungen, für die sich der Forscher entscheidet. Auch unüberwachte Verfahren erfordern nämlich eine Kalibrierung bei LDA ist beispielsweise die Variable $K$ von großer Bedeutung (vgl. Jacobi et al. 2016, S. 100). Sie gibt die Anzahl der Topic-Cluster an, die gebildet werden sollen - sie entscheidet also maßgeblich über die „Auflösung“ der Topics - ein größeres $K$ entspricht einer höheren Granularität.

Eine weitere zentrale, bisher wenig beachtete Herausforderung im Zusammenhang mit LDA ist die Reliabilität der generierten Modelle. Da es sich um ein probabilistisches Verfahren handelt, erzeugen gleiche Variablenkonstellationen abweichende Cluster. Beide zentralen Herausforderungen wurden im Zuge dieser Arbeit berücksichtigt (s. unten).

Analysen mit LDA zielen oft auf die bloße Beschreibung von Themenverteilungen in Korpora. Die Modelle bieten jedoch durchaus weiterführende Möglichkeiten der Analyse (vgl. Puschmann und Scheffler 2016, S. 13). Die Interpretation der LDAModelle als Indikatoren für interne Vielfalt ist in diesem Sinne ein Beispiel dafür, wie sich durch Topic Modeling Erkenntnisse über Themenverteilungen hinaus generieren lassen.

\subsection{Vielfaltsmessung mittels LDA}

Durch das Topic-Clustering-Verfahren wird jedem Wort (gemeint sind token) eines Korpus genau ein Thema zugeordnet. Zusätzlich ist jedem Wort im Falle dieser Untersuchung eine Partei zugeordnet - je nachdem, welcher Partei der Politiker angehört, der den zum Text gehörigen Link gepostet hat (wurde ein Text von Politikern unterschiedlicher Parteien verlinkt, wurden den Worten des Artikels mehrere Parteien zugewiesen). Es lässt sich also der Anteil jeder Partei an jedem Topic feststellen. Hierbei ist es natürlich möglich, dass eine Partei ein Topic stark dominiert, weil Politiker der Partei besonders viel zu diesem Thema gepostet haben, oder dass ein Topic von allen Parteien gleichermaßen bedient wird, und daher eine gleichmäßige Durchmischung aufweist. Um nun mathematisch zu beschreiben, wie vielfältig die in den Verlinkungen gespiegelte Themenagenda einer Partei ist, sind wir folgendermaßen vorgegangen:

- Normierung

Um eine Vergleichbarkeit von Topics und Parteien untereinander zu gewährleistet, muss verhindert werden, dass die Größe der Topics das Ergebnis beeinflusst. Gleiches gilt für die absolute Anzahl der Wörter, die einer Partei zugeordnet wurden. Wir haben die absoluten Werte daher so normiert, dass die Summe eines jedes Topics 1 ergibt, d.h. jedes Topic erhält dasselbe Gewicht. Anschließend haben wir mit der Summe der Wort-Partei-Zuordnungen über alle Topics normiert, sodass die absolute Anzahl der Wörter, die einer Partei zugeordnet werden, die Interpretation nicht verzerrt. Die resultierende Matrix kann als Heatmap dargestellt werden: Hier wird die relative Anzahl der Zuordnungen von Wörtern aus Partei-Artikeln zu einem Topic dargestellt (siehe Abb. 2). Diese Darstellung ermöglicht Vergleiche von Topics innerhalb einer Partei und von Parteien innerhalb eines Topics. 


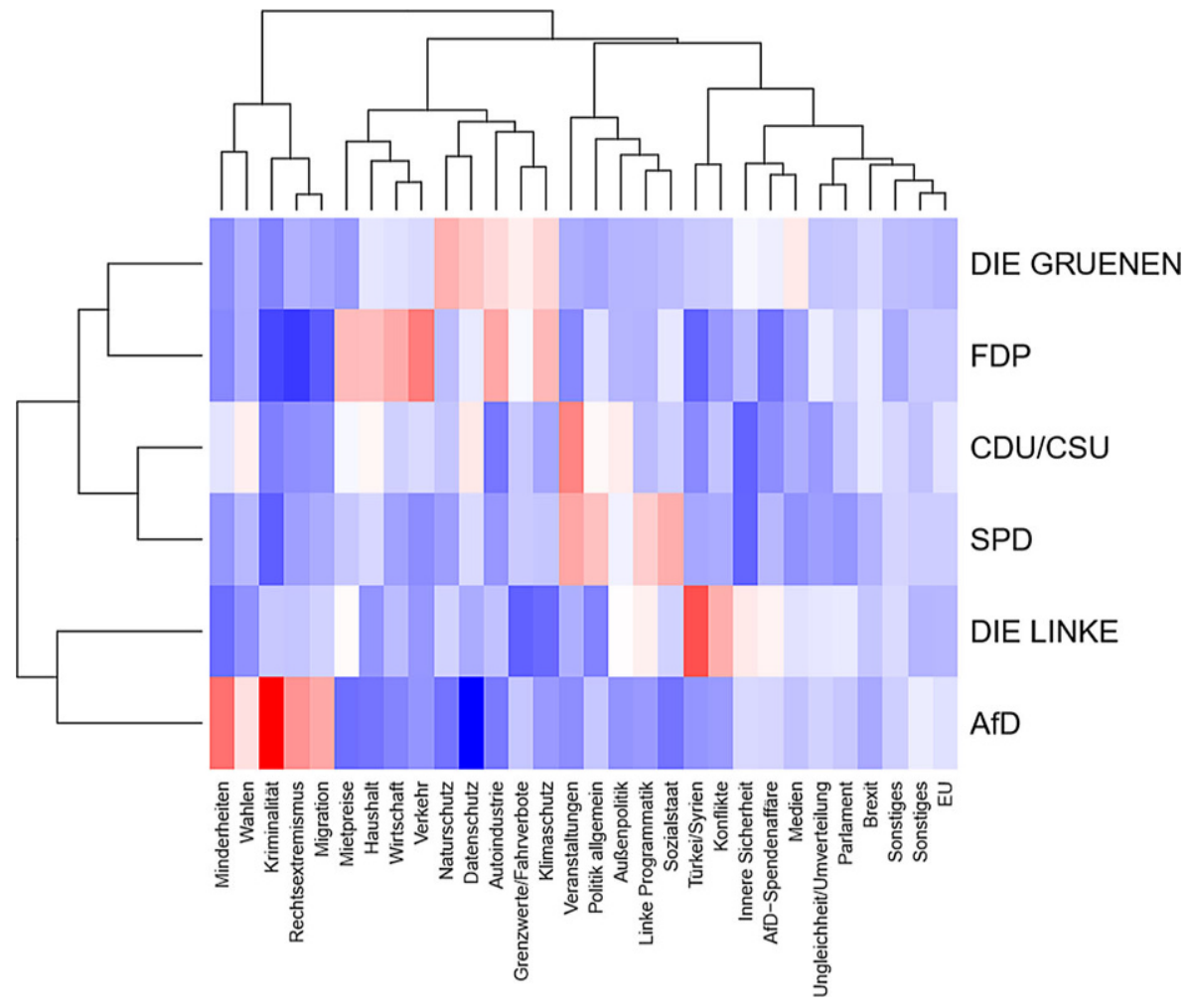

Abb. 2 Heatmap der auf Topicsumme und Fraktionssumme normierten Topiczuordnungen pro Fraktion für die Protoypen-LDA mit $\mathrm{K}=30$ und $\alpha=\eta=1 / 30$. Je intensiver die rötliche Färbung einer Zelle ist, desto stärker ausgeprägt ist die Fokussierung dieser Fraktion auf dieses Topic, wohingegen intensivere Blautöne für Vernachlässigung dieser Themen im Vergleich zu anderen Fraktionen stehen. Die Topics bzw. Fraktionen wurden hierarchisch mit Complete Linkage auf den Distanzen der normierten Topiczuordnungen geclustert (vgl. Hastie et al. 2009, S. 520-525). Das heißt zum Beispiel, dass die AfD und Die Linke sich näher sind als die AfD bzw. Die Linke der unähnlichsten Fraktion aus dem Cluster aus SPD, CDU/CSU, FDP, Die Grünen. Topic-Überschriften basieren auf einer Interpretation von Top-Words (den repräsentativsten Wörtern eines Topics) und Top-Artikeln (den repräsentativsten Texten)

\section{- Gini-Koeffizient}

Thematische Vielfalt wird in dieser Untersuchung aus zwei Perspektiven analysiert: Ausgehend von dem einzelnen Topic wird die Frage beantwortet, wie gleichmäßig es sich über die Parteien verteilt, und ausgehend von der einzelnen Partei wird betrachtet, wie gleichmäßig sie die Themen adressiert. Sowohl die Vielfalt eines Topics über die Parteien hinweg, wie auch die Gleichmäßigkeit der Themenadressierung durch eine Partei können mit dem Gini-Koeffizienten (vgl. Hartung et al. 2009: 52-53) berechnet werden. Der Gini-Koeffizient ist ein statistisches $\mathrm{Ma} \beta$ für Ungleichverteilungen und nimmt Werte im Bereich $(0,1)$ an, wobei 0 für eine perfekte Gleichverteilung steht - in diesem Fall ein Topic, das von allen Parteien gleichermaßen adressiert wird beziehungsweise aus Sicht der Parteien: eine Partei, die zu allen Topics im selben Umfang twittert. Höhere Werte indizieren ei- 
ne höhere Heterogenität der Agenda einer Partei beziehungsweise ein gesteigertes Ungleichgewicht der Aufteilung eines Themas auf die untersuchten Parteien.

Um die Reliabilität der Vielfaltsmessung zu gewährleisten - also modellunabhängige Inferenzen - haben wir die Gini-Koeffizienten auf LDAs mit 56 verschiedenen Variablenkonstellationen nach Griffiths und Steyvers (2004) gerechnet. Der Hypothesentest (H1) ist also unabhängig von der Betrachtung einzelner Modelle möglich.

\subsection{LDA-Prototyp}

Zur qualitativen Interpretation einzelner LDAs (vgl. Koppers et al. 2019) haben wir für ausgesuchte Variablenkonstellationen zusätzlich einen LDA-Prototypen gebildet (R-Paket ldaPrototype, vgl. Rieger 2020) - so erhöhen wir die Reliabilität des zu interpretierenden Modells (vgl. Rieger et al. 2020). Vergleichbar mit Niekler und Jähnichen (2012) berechnen wir hierbei im ersten Schritt eine Ähnlichkeit der Topics verschiedener Modelle zueinander (mittels eines modifizierten Jaccard-Koeffizienten). Hieraus lässt sich ein Reliabilitätskoeffizient (beispielsweise einer bestimmten Variablenkonstellation) ableiten, indem eine durchschnittliche Ähnlichkeit aller Topics zueinander angegeben wird (vgl. Maier et al. 2018, S. 97). Zur Berechnung des LDA-Prototypen gehen wir einen Schritt weiter, indem wir dasjenige Modell herausgreifen, dessen Topics die größte Ähnlichkeit zu den Topics aller anderen Modelle aufweist. Der LDA-Prototyp ist dann also das Modell mit dem größten Ähnlichkeitswert über alle Paarungen. So wird verhindert, dass das zu interpretierende Modell eine extreme beziehungsweise instabile Ausprägung einer bestimmten Variablenkonstellation darstellt.

\subsection{Topics mit hohem Engagement}

Um die zweite Hypothese zu prüfen, haben wir berechnet, wie viel Engagement einzelne Themen auf Twitter auslösen - also welche Themen nach den Relevanzindikatoren der Plattform besonders relevant sind. Hierfür haben wir für jeden Text einen Engagement-Wert berechnet, der sich aus der Summe von Retweets und Likes des zugrundeliegenden Tweets ergibt. Dieser Wert lässt sich für jeden von einer Partei geteilten Text berechnen. Wir haben nun anhand der Topicverteilung eines jeden Textes die latenten Engagement-Werte für jedes Topic in jedem Text ermittelt. Auf Basis dieser Kennwerte lassen sich durch Normierung mit der Anzahl der zugehörigen Tweets die mittleren Engagement-Werte pro Partei und Topic berechnen. Der Wert gibt also an, wie viel Engagement eine Partei in den entsprechenden Topics pro geteiltem Text generiert. Mit anderen Worten: Der Wert lässt eine Aussage darüber zu, wie viel Engagement ein Thema auslöst, wenn es von Politikern einer bestimmten Partei adressiert wird. Setzt man diesen Wert ins Verhältnis zu der Themenverteilung pro Partei, wird deutlich, inwiefern die Themen, die von den Politikern adressiert werden, auch diejenigen sind, die Engagement auslösen. Über diese Korrelation wird das Maß an Synergie zwischen sozialem und technologischem Gatekeeping beschrieben (H2). Auch hier wird durch mehrfache Berechnung (für jedes LDA-Modell) Reliabilität gewährleistet. 


\section{Resultate}

H1 Im Vergleich zur Verlinkungspraxis der anderen Parteien auf Twitter spiegelt sich in den von der AfD verlinkten Texten eine weniger vielfältige Themen-Agenda.

Betrachtet man die Durchmischung der Themen, lassen sich zunächst grob zwei Kategorien von Topics unterscheiden: Cluster, die deutlich von einer (oder von wenigen) Parteien dominiert werden (mit einem hohen Gini-Koeffizienten $>0,2$ ) und Themen, die eine relativ gleichmäßige Durchmischung aufweisen (mit einem kleinen Gini-Koeffizienten $<0,1$ ). Für ein Modell mit 30 Topics $^{12}$ (siehe Abb. 1) ergibt sich nach dieser Klassifizierung ein Verhältnis von 11 homogeneren Querschnittsthemen zu 19 Themen, bei denen nur 1-2 Parteien einen inhaltlichen Schwerpunkt setzen. Insbesondere der Blick auf die Themen der letztgenannten Kategorie verdeutlicht die inhaltliche Nähe der Verlinkungspraxis zur jeweiligen politischen Programmatik. So gibt es Expertentopics, die eindeutig von einer bestimmten Partei bedient werden: Die Grünen besetzen beispielsweise die Themenbereiche Daten- und Naturschutz; FDP-Politiker posten intensiv zu Themen wie Verkehr und Wirtschaft; Politiker der Linken posten vermehrt zu internationalen Konflikten. Neben diesen durch IssueOwnership charakterisierten Topics gibt es Themenbereiche, in denen eine deutliche Polarisierung zwischen zwei Parteien sichtbar wird: Beispielsweise dominieren Grüne und FDP in den umstrittenen Bereichen Klimaschutz und Autoindustrie (in der Heatmap sind die Themen für beide Parteien rötlich eingefärbt) - die Parteien stehen sich daher auch thematisch nahe.

Neben diesen Topics, die von zwei diametral positionierten Parteien dominiert werden, gibt es solche, in denen die Regierung kritisch thematisiert wird, besonders deutlich im Topic Innere Sicherheit (Top-Words z. B. Anfrage, Untersuchungsausschuss oder Amri) - hier äußern sich alle Parteien bis auf die Regierungsfraktionen. $\mathrm{Zu}$ den Themen, zu denen sich alle Parteien gleichermaßen äußern, gehören der Brexit oder die EU (mit sehr kleinem Gini-Koeffizient, gleichmäßige Einfärbung in der Heatmap).

Die rechtspopulistische AfD nimmt eine Sonderrolle ein: Die vier Themen, die am deutlichsten durch eine Partei besetzt werden und daher auch den höchsten GiniKoeffizienten aufweisen, sind populistische Kernthemen, die von der AfD bedient werden (unten links in der Heatmap - in geringer thematischer Distanz: Migration, Kriminalität, Rechtsextremismus ${ }^{13}$ und Minderheiten). Gleichzeitig spielt die AfD

\footnotetext{
12 Während über die Hypothesen weitgehend modellunabhängig entschieden werden kann (durch den Vergleich mehrerer Modelle), ist eine qualitative Auswertung nur auf Basis einer - hier qualitativ nach Interpretierbarkeit gewählten - Granularitätsstufe sinnvoll, also mit Hilfe des Prototyps einer Variablenkonstellation. Hierdurch ergeben sich die für die LDA typischen Validitätsprobleme, denen hier mit den üblichen qualitativen Schritten begegnet wurde (siehe semantische Intra-Topic-Validität, vgl. Maier et al. 2018, S. 99). Die bekannten Unschärfen dieser Validierung haben indes keine Konsequenzen für die Prüfung der Hypothesen.

${ }^{13}$ Dieses Thema wird im Sinne einer Strategie der doppelten Differenzierung (Groshek und Engelbert 2013) instrumentalisiert: Die AfD-Politiker empören sich hier über den Vorwurf der rechten Ideologie (Abgrenzung zum Extremismus) und bekunden gleichzeitig Sympathien für rechte Demonstrationen etc. (Abgrenzung zur Elite).
} 

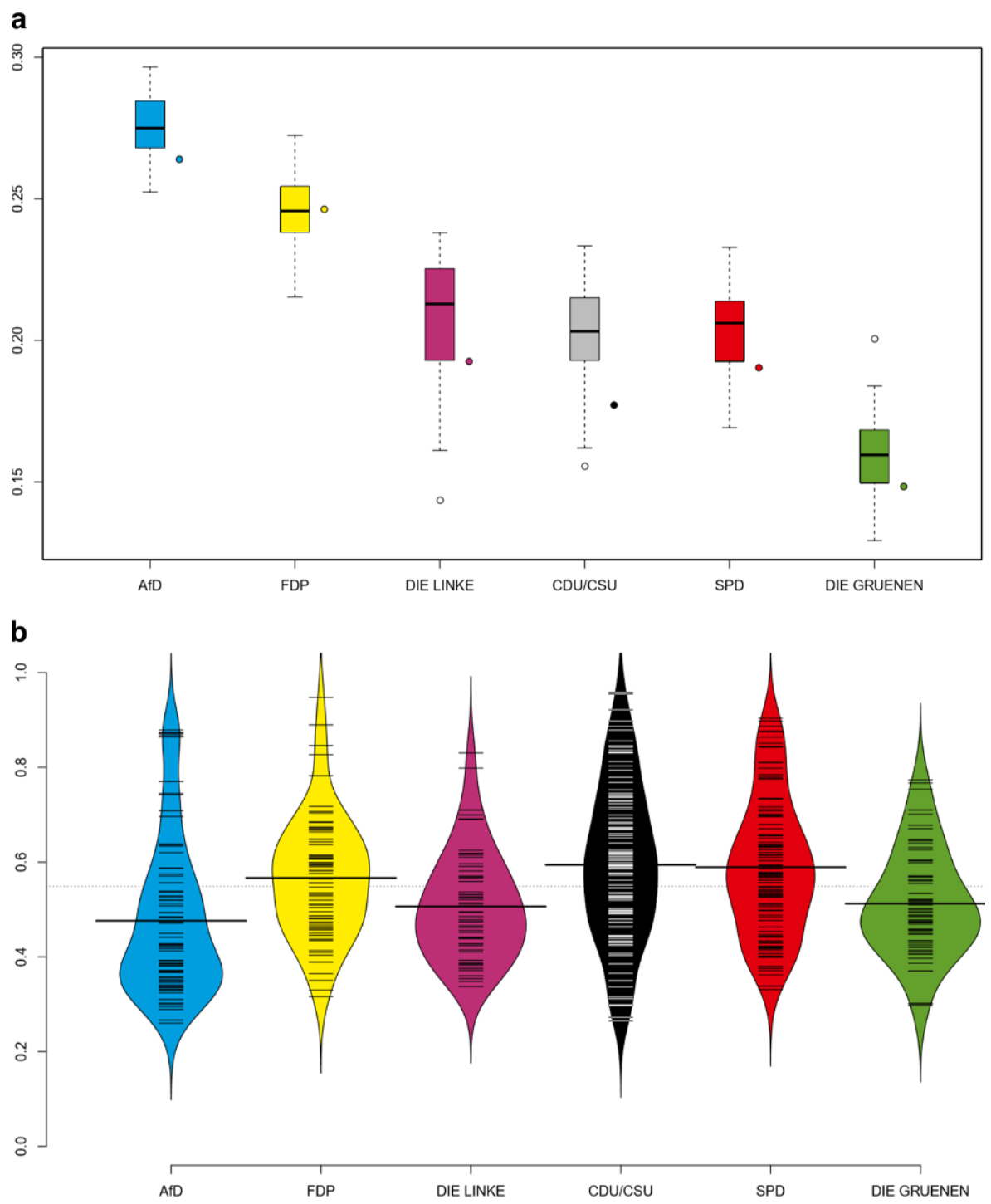

Abb. 3 a Jeweils ein Boxplot der Verteilung der Gini-Koeffizienten der entsprechenden Fraktion über 56 verschiedene Parameterkombinationen für die LDA $(K=20, \ldots, 75, \alpha=\eta=1 / K)$. Der farblich gekennzeichnete Punkt gibt den Gini-Koeffizienten der jeweiligen Fraktion in der betrachteten Prototypen-LDA mit $K=30$ und $\alpha=\eta=1 / 30$ wieder. b Beanplots der Verteilungen der Gini-Koeffizienten pro Twitterer der untersuchten Fraktionen im Bundestag für die Protoypen-LDA mit $K=30$ und $\alpha=\eta=1 / 30$

in vielen Themenbereichen keine Rolle, beispielsweise in den Bereichen Daten-, Klima- und Umweltschutz.

Die AfD ist damit eindeutig die Partei, deren thematisches Engagement sich am wenigsten gleichmäßig über die Palette der Topics verteilt. Mit anderen Worten: Die Themenpalette der AfD weist deutlich weniger Vielfalt auf als die der anderen 
Parteien. Diese Inferenz lässt sich modellunabhängig bestätigen. Bei jeder der gerechneten 56 Variablenkonstellation (dargestellt durch den Boxplot, siehe Abb. 3) ergibt sich diese Sonderstellung der AfD. Vergleicht man nun die durchschnittliche Vielfalt der Posts der einzelnen Politiker einer Partei (in Abb. 3 durch Beans dargestellt), wird deutlich, dass auch hier die AfD eine exponierte Position einnimmt: Die AfD-Politiker twittern, betrachtet auf der Akteursebene, verhältnismäßig heterogen (resultierend in einem niedrigen Gini-Koeffizienten). Sie sind thematische Generalisten in dem Sinne, dass die meisten von ihnen mehrere Kernthemen der populistischen Partei bedienen. Gleichzeitig ist der Gini-Koeffizient der Partei der mit Abstand höchste (visualisiert durch die Boxplots in Abb. 3) - die Agenda der AfD konzentriert sich, wie oben beschrieben, auf wenige Themen und ist damit wenig vielfältig in Relation zu den anderen Parteien.

Anders sieht es beispielsweise bei den sich thematisch nahestehenden Koalitionsparteien SPD und CDU/CSU aus: Sie gehören zu den Parteien mit den heterogensten, vielfältigsten Themenprofilen (resultierend in einem niedrigen Gini-Koeffizienten). Diese entstehen auf individueller Ebene durch das Zusammenwirken unterschiedlicher thematischer Spezialisten, die vor allem zu ihrem jeweiligen Thema twittern: Die Analyse von SPD und CDU/CSU ergibt also im Aggregat einen niedrigen (dargestellt durch den Boxplot in Abb. 3), auf individueller Ebene einen hohen GiniKoeffizienten (dargestellt durch den Beanplot in Abb. 3). Interessanterweise ist bei der FDP ein ähnlicher Spezialisierungsgrad wie bei den Koalitionsparteien zu beobachten; da die Experten aber unter sich homogener sind, ist die thematische Vielfalt im Aggregat niedriger als bei den Koalitionsparteien. Bei Linken und Grünen ist der Spezialisierungsgrad der Politiker weniger stark ausgeprägt, was sich hier in einer breiteren individuellen Themenagenda spiegelt. Insbesondere Die Grünen stechen hervor; sie adressieren von allen Parteien die vielfältigste Palette an Themen.

H2 Themen, die von der AfD besetzt werden, sind - gemessen an EngagementMetriken (Likes, Shares) - erfolgreicher als Themen anderer Parteien auf Twitter.

Posts der AfD-Bundestagsabgeordneten lösen mehr Engagement aus als die der anderen Parlamentarier. Das gilt für die Gesamtheit der Tweets und noch deutlicher für Tweets im untersuchten Datensatz (also Posts, in denen auf andere Seiten verlinkt wird) - absolut für alle Tweets in Summe und auch relativ für das mittlere Engagement pro Tweet. Betrachtet man die LDA mit 30 Topics, sind es Posts der AfD zu Themen wie Kriminalität, Rechtsextremismus, Migration, Minderheiten und Ungleichheit/Umverteilung, die (sowohl relativ als auch absolut gesehen) am meisten Engagement auslösen. Keine Partei schafft es mit ihren Tweets, ähnlich viele Likes und Retweets zu evozieren. Nach der AfD folgen mit weitem Abstand Die Linke (Themen mit dem größten mittleren Engagement: Ungleichheit/Umverteilung, Türkei/Syrien), Die Grünen (Rechtsextremismus, Klimaschutz), SPD (Rechtsextremismus, linke Programmatik), FDP (Klimaschutz, Haushalt) und die Union (Rechtsextremismus, Wahlen).

Des Weiteren haben wir untersucht, ob der Engagement-Erfolg einzelner Themen mit den Selektionsentscheidungen der Politiker korreliert. Wir haben also den Zusammenhang analysiert zwischen dem metrisch quantifizierbaren Erfolg von Tweets zu einem Thema und der Menge der Posts, die zu ebendiesem Thema von einer 


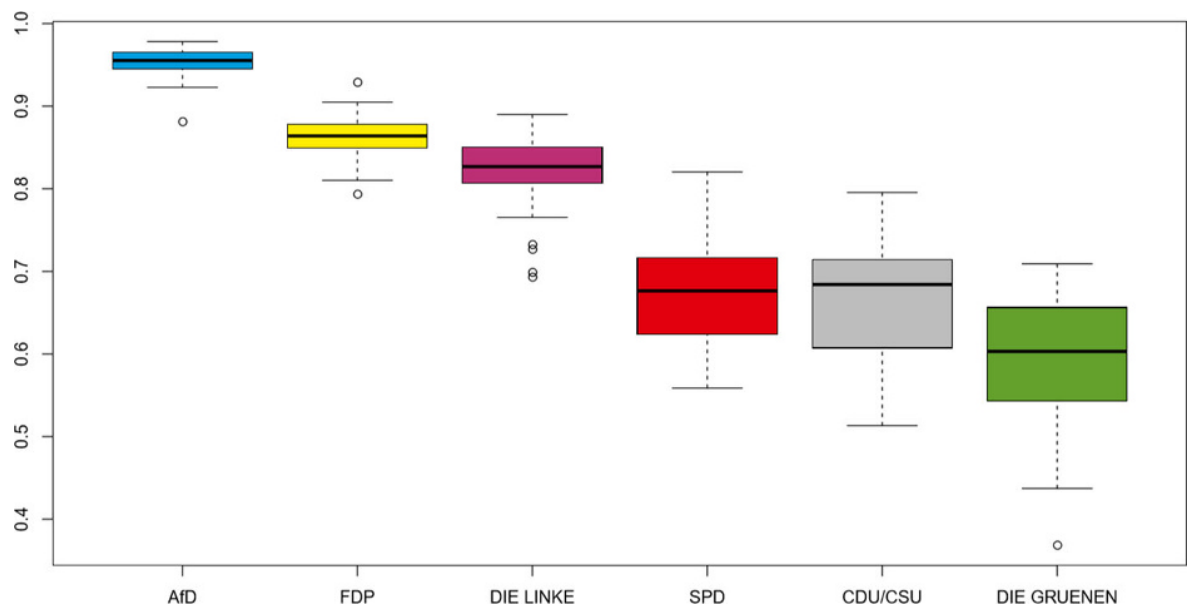

Abb. 4 Korrelation des Engagement-Scores pro Thema und Partei mit der absoluten Anzahl an Topiczuordnungen pro Partei (Rangkorrelationskoeffizient nach Spearman) - für insgesamt 56 verschiedene Parameterkombinationen $(K=20, \ldots, 75, \alpha=\eta=1 / K)$

Partei veröffentlicht wurden. Hierbei zeigt sich, dass die Korrelation zwischen der Themensetzung durch die verlinkten Texte und dem Engagement-Erfolg der zugehörigen Tweets bei keiner der anderen Parteien so deutlich ist wie bei der AfD (siehe Abb. 4).

\section{Fazit}

Ziel dieser Untersuchung war es zum einen, die Verlinkungspraxis populistischer Akteure auf Basis repräsentativer Daten zu beschreiben. Zum anderen sollte das synergetische Zusammenwirken aus Engagement-Metriken und populistischer Themensetzung offengelegt werden. Hierzu haben wir 56.107 Texte analysiert, die von Bundestagsabgeordneten in der laufenden Legislaturperiode in Tweets geteilt wurden. Die zentralen Ergebnisse der Untersuchung sind:

1. Die Themenagenda rechtspopulistischer Akteure (hier am Beispiel der AfD) unterscheidet sich deutlich von den Themenprioritäten anderer Parteien. Sie weist eine andere Struktur auf - wenige Themen werden intensiv adressiert - und umfasst andere inhaltliche Schwerpunkte (s. unten). Hypothese 1 kann somit als bestätigt angesehen werden: Im Vergleich zu den Verlinkungen der anderen Parteien auf Twitter spiegelt sich in denen der AfD eine weniger vielfältige Themen-Agenda - dieses Ergebnis konnte für jede der gerechneten LDA-Variablenkonstellationen bestätigt werden.

Das Mittel des Teilens journalistischer Inhalte ist offenbar zentral für die populistische Kommunikationsstrategie: Während die AfD-Poltiker auf Twitter ansonsten nicht aktiver sind als andere Parlamentarier, stechen sie beim Sharing deutlich hervor. 
2. Die Themenselektion der populistischen Akteure korreliert stärker mit dem metrischen Erfolg einzelner Themen, als dies bei anderen Parteien der Fall ist - ein synergetisches Verhältnis konnte hier also deutlich nachgewiesen werden. Auch dieses Ergebnis ergab sich gleichermaßen für jede der gerechneten LDA-Variablenkonstellationen. Hypothese 2 wurde hiermit bestätigt: Themen, die von der AfD besetzt werden, sind - gemessen an Engagement-Metriken (Likes, Shares) erfolgreicher als die Themen anderer Parteien auf Twitter.

Die Themen, die in der Literatur als charakteristisch für Populisten beschrieben werden (vgl. Ernst et al. 2018, S. 172), spiegeln sich in den Themen, mit denen AfD-Politiker am meisten Engagement auslösen: Migration, die Verhandlung von Identität in der Auseinandersetzung mit Rechtsextremismus und Minderheiten (also outgroups), Kriminalität, also Korruption und Verbrechen, sowie die Thematisierung wirtschaftlicher Not, insbesondere über Chancen- und Verteilungsfragen. Einzig der in der Literatur als typisch beschriebene Themenkomplex der europäischen Integration spielt im untersuchten Fall eine untergeordnete Rolle.

Es konnten des Weiteren keine Indizien für die These einer inhaltlichen Expansion (vgl. Krämer 2017) über die typisch populistischen Themenbereiche hinaus nachgewiesen werden. Allerdings würde die strategische Umsetzung des Anspruchs der neuen ,Volkspartei“, die sukzessive Erschließung neuer Themenbereiche, erst durch eine longitudinale Analyse sichtbar. Die vorliegende Studie bietet hierfür einen geeigneten Ausgangspunkt.

In einem nächsten Schritt wäre beispielsweise zu fragen, wie die Verzerrung der Medienagenda durch das populistische Gatekeeping auf den Journalismus zurückwirkt - beispielsweise vermittelt durch eine redaktionelle Orientierung an den Relevanzindikatoren der Plattformen (vgl. Caplan und Boyd 2018, S. 5-8). Des Weiteren wäre es naheliegend, die Entstehung der Engagement-Agenda-Korrelation qualitativ oder longitudinal quantitativ zu beleuchten: Agieren AfD-Politiker strategisch, indem sie erfolgsversprechende Themen besetzen? Gibt es also ein Wechselspiel zwischen Themensetzung und metrischem Erfolg? Bedienen sie bewusst die Identität eines bestimmten Kollektivs?

Aus methodischer Perspektive hat sich bestätigt, dass die Analyse externer Vielfalt $\mathrm{zu}$ anderen Inferenzen führt als eine Betrachtung der internen Vielfalt. Würde man allein die Vielfalt verlinkter Medien vergleichen, käme man zum Schluss, dass sich das Gatekeeping der AfD nicht deutlich von dem der anderen Parteien unterscheidet. Der methodische Schwerpunkt auf externe Vielfalt könnte so zu einer Normalisierung populistischer Kommunikation beitragen. Der Mehrwert einer Analyse auf Inhaltsebene - statt nur auf Quellenebene - wird hier evident. Die Kontrastierung beider Ebenen ermöglicht darüber hinaus, die Beobachtung der Paradoxie populistischer Kommunikation (vgl. Haller und Holt 2019) auf die Ebene des Gatekeepings zu übertragen: Die Verlinkung tradierter journalistischer Quellen verleiht der dahinterstehenden populistischen Agenda Seriosität. Diese unterstützt als Zerrspiegel der journalistischen Agenda paradoxerweise gleichzeitig die populistische Kritik an journalistischer Themensetzung.

Auch in Hinblick auf die anderen Parteien liefert die vorliegende Arbeit interessante Erkenntnisse: Besonders frappierend ist die Ähnlichkeit der Koalitionspar- 
teien in allen gemessenen Dimensionen. Hiermit werden frühere Studien zur inhaltlichen Nähe der Regierungsparteien bestätigt (vgl. Caton et al. 2015, S. 8; Tromble 2016, S. 679). Bei den Oppositionsparteien stechen FDP und Die Grünen hervor. Ähnlich wie die AfD-Parlamentarier verlinken Politiker der FDP vor allem Texte zu bestimmten Kernthemen, die Engagement auslösen. Inhaltlich unterscheiden sich diese indes stark von den Themen der Rechtspopulisten: Bundestagsabgeordnete der FDP konzentrieren sich zum einen auf Expertentopics im ökonomischen Bereich und zum anderen auf polarisierende Themen, bei denen sie oft eine Gegenposition zu den Grünen einnehmen.

Analog zu früheren Studien (vgl. Caton et al. 2015, S. 14) kann bestätigt werden, dass sich besonders das Social-Media-Verhalten der Grünen von dem der anderen Parteien unterscheidet. Sie stellen in mehrerlei Hinsicht das Gegenmodell zur AfD dar: Sie haben die vielfältigste Themenagenda und ihre Themenselektion korreliert am wenigsten mit dem Engagement der User. Während die AfD durch die issueownership typisch populistischer Themen und eine damit verbundene Vielfaltsreduktion hervorsticht, kommunizieren die Grünen vergleichsweise vielfältig.

Die vorgelegten Ergebnisse sind insbesondere in zweierlei Hinsicht limitiert: Sie beschränken sich auf die Plattform Twitter, die zwar für Elitenkommunikation relevant ist, jedoch nur beschränkt für die Kommunikation zwischen Politikern und Wählerschaft. Politiker erreichen auf anderen Plattformen, insbesondere Facebook, eine größere Zielgruppe. Es wäre daher zu prüfen, ob sich die Ergebnisse dieser Studie in einem Cross-Plattform-Vergleich bestätigen ließen. Außerdem beschränkt sich die Analyse auf Texte, zu denen der Scraper Zugriff hatte - Multimedia-Inhalte wurden in der Analyse nicht berücksichtigt, genauso fehlen Inhalte von Facebook. In einem nächsten Schritt wäre es daher beispielsweise wünschenswert, das Korpus um Social-Media-Posts und transkribierte Video- und Audioinhalte zu ergänzen.

Funding Open Access funding provided by Projekt DEAL.

Open Access Dieser Artikel wird unter der Creative Commons Namensnennung 4.0 International Lizenz veröffentlicht, welche die Nutzung, Vervielfältigung, Bearbeitung, Verbreitung und Wiedergabe in jeglichem Medium und Format erlaubt, sofern Sie den/die ursprünglichen Autor(en) und die Quelle ordnungsgemäß nennen, einen Link zur Creative Commons Lizenz beifügen und angeben, ob Änderungen vorgenommen wurden.

Die in diesem Artikel enthaltenen Bilder und sonstiges Drittmaterial unterliegen ebenfalls der genannten Creative Commons Lizenz, sofern sich aus der Abbildungslegende nichts anderes ergibt. Sofern das betreffende Material nicht unter der genannten Creative Commons Lizenz steht und die betreffende Handlung nicht nach gesetzlichen Vorschriften erlaubt ist, ist für die oben aufgeführten Weiterverwendungen des Materials die Einwilligung des jeweiligen Rechteinhabers einzuholen.

Weitere Details zur Lizenz entnehmen Sie bitte der Lizenzinformation auf http://creativecommons.org/ licenses/by/4.0/deed.de.

\section{Literatur}

Bechmann, A., \& Nielbo, K.L. (2018). Are we exposed to the same "news" in the news feed? Digital Journalism, 6, 990-1002. 
Blei, D. M., Ng, A. Y., \& Jordan, M.I. (2003). Latent Dirichlet allocation. Journal of machine learning research, 3, 993-1022.

Caplan, R., \& d, B. (2018). Isomorphism through algorithms: Institutional dependencies in the case of Facebook. Big Data \& Society, 5(1), 205395171875725.

Caton, S., Hall, M., \& Weinhardt, C. (2015). How do politicians use Facebook? An applied Social Observatory. Big Data \& Society, 2(2), 1-18.

Diffbot. (2019). Mission. https://www.diffbot.com/company/. Zugegriffen: 25. Okt. 2019.

Dittrich, P.-J. (2017). Social Networks and Populism in the EU. Four Things you should know. Policy Paper, 19(2), 1-20. https://www.delorsinstitut.de/2015/wp-content/uploads/2017/04/20170419_ SocialNetworksandPopulism-Dittrich.pdf. Zugegriffen: 25. Okt. 2019.

Dvir-Gvirsman, S. (2017). Media audience homophily: Partisan websites, audience identity and polarization processes. New Media \& Society, 19, 1072-1091.

Engesser, S., Ernst, N., Esser, F., \& Büchel, F. (2017). Populism and social media: how politicians spread a fragmented ideology. Information, Communication \& Society, 20, 1109-1126.

Ernst, N., Esser, F., Blassnig, S., \& Engesser, S. (2018). Favorable Opportunity Structures for Populist Communication: Comparing Different Types of Politicians and Issues in Social Media, Television and the Press. The International Journal of Press/Politics, 24, 165-188.

Freeden, M. (2008). Ideologies and political theory: a conceptual approach (Reprinted). Oxford: Clarendon Press.

Fuchs, M. (2019). mdb-bundestag. https://twitter.com/wahl_beobachter/lists/mdb-bundestag. Zugegriffen: 25. Okt. 2019.

Gerbaudo, P. (2018). Social media and populism: an elective affinity? Media, Culture \& Society, 40, $745-753$.

Griffiths, T. L., \& Steyvers, M. (2004). Finding scientific topics. Proceedings of the National Academy of Sciences, 101(Supplement 1), 5228-5235.

Groshek, J., \& Engelbert, J. (2013). Double differentiation in a cross-national comparison of populist political movements and online media uses in the United States and the Netherlands. New Media \& Society, 15, 183-202.

Hagen, L. M. (1992). Die opportunen Zeugen. Konstruktionsmechanismen von Bias in der Zeitungsberichterstattung über die Volkszählungsdiskussion. Publizistik, 37, 444-460.

Haller, A., \& Holt, K. (2019). Paradoxical populism: how PEGIDA relates to mainstream and alternative media. Information, Communication \& Society, 22:12, 1665-1680.

Hartung, J., Elpelt, B., \& Klösener, K.-H. (2009). Statistik - Lehr- und Handbuch der angewandten Statistik (15. Aufl.). München: Oldenbourg Wissenschaftsverlag.

Hastie, T., Tibshirani, R., \& Friedman, J. (2009). The Elements of Statistical Learning: Data Mining,Inference, and Prediction (2. Aufl.). New York: Springer.

Ihm, J., \& Kim, E. (2018). The hidden side of news diffusion: Understanding online news sharing as an interpersonal behavior. New Media \& Society, 2(0), 4346-4365.

Jacobi, C., van Atteveldt, W., \& Welbers, K. (2016). Quantitative analysis of large amounts of journalistic texts using topic modelling. Digital Journalism, 4(1), 89-106.

Kearney, M.W. (2019). rtweet: Collecting Twitter Data (Version 0.6.9). https://cran.r-project.org/ package=rtweet. Zugegriffen: 25. Okt. 2019.

Keyling, T. (2017). Kollektives Gatekeeping. Wiesbaden: Springer.

Koppers, L., Rieger, J., Boczek, K., \& von Nordheim, G. (2019). tosca: Tools for Statistical Content Analysis (Version 0.1-4). https://CRAN.R-project.org/package=tosca. Zugegriffen: 25. Okt. 2019.

Krämer, B. (2017). Populist online practices: the function of the Internet in right-wing populism. Information, Communication \& Society, 20, 1293-1309.

Maier, D., Waldherr, A., Miltner, P., Wiedemann, G., Niekler, A., Keinert, A., ... Adam, S. (2018). Applying LDA Topic Modeling in Communication Research: Toward a Valid and Reliable Methodology. Communication Methods and Measures, 12(2-3), 93-118.

May, P. (2019). „Wir haben uns als Volkspartei etabliert“. https://www.deutschlandfunk.de/joergmeuthen-afd-wir-haben-uns-als-volkspartei-etabliert.694.de.html?dram:article_id=457774 Zugegriffen: 25. Okt. 2019.

McQuail, D., \& Van Cuilenburg, J. J. (1983). Diversity as a Media Policy Goal: A Strategy for Evaluative Research and a Netherlands Case Study. Gazette, 31(3), 145-162.

Mudde, C. (2004). The Populist Zeitgeist. Government and Opposition, 39, 541-563.

Napoli, P. M. (1999). Deconstructing the Diversity Principle. Journal of Communication, 49(4), 7-34.

Niekler, A., \& Jähnichen, P. (2012). Matching results of latent dirichlet allocation for text. Proceedings of the 11th International Conference on Cognitive Modeling (ICCM 2012), 317-322. 
Nuernbergk, C., \& Conrad, J. (2016). Conversations and Campaign Dynamics in a Hybrid Media Environment: Use of Twitter by Members of the German Bundestag. Social Media \& Society, 2, 1-14.

Octoparse. (2019). Easy Web Scraping for Anyone. https://www.octoparse.com/. Zugegriffen: 25. Okt. 2019.

Oelsner, K., \& Heimrich, L. (2015). Social Media Use of German Politicians: Towards Dialogic Voter Relations? German Politics, 24, 451-468.

Ooms, J., \& Hester, J. (2019). spelling: Tools for Spell Checking in R. https://cran.r-project.org/web/ packages/spelling/index.html. Zugegriffen: 25. Okt. 2019.

Petrocik, J.R. (1996). Issue Ownership in Presidential Elections, with a 1980 Case Study. American Journal of Political Science, 40, 825-850.

Puschmann, C., \& Scheffler, T. (2016). Topic Modeling for Media and Communication Research: A Short Primer. HIIG discussion paper series 5. 1-17.

Rieger, J. (2020). ldaPrototype: Prototype of Multiple Latent Dirichlet Allocation Runs. R package version 0.1.1. https://CRAN.R-project.org/package=ldaPrototype. Zugegriffen: 10. März 2020.

Rieger, J., Koppers, L., Jentsch, C., \& Rahnenführer, J. (2020). Improving Reliability of Latent Dirichlet Allocation by Assessing Its Stability Using Clustering Techniques on Replicated Runs. http://arxiv. org/abs/2003.04980. Zugegriffen: 10. März 2020.

Rooduijn, M., van Kessel, S., Froio, C., Pirro, A., De Lange, S., Halikiopoulou, D., Lewis, P., Mudde, C., \& Taggart, P. (2019). The PopuList: An overview of populist, far right, far left and Eurosceptic parties in Europe. https://popu-list.org. Zugegriffen: Data set.

Saalfeld, T., \& Dobmeier, R. (2012). The Bundestag a nd German Citizens: More Communication, Growing Distance. The Journal of Legislative Studies, 18, 314-333.

Schmidt, J.-H. (2017). Twitter-Nutzung von Kandidierenden der Bundestagswahl 2017. Verbreitung, Aktivität und Informationsquellen. Media Perspektiven, o. J.(12), 616-629.

Singer, J. B. (2013). User-generated visibility: Secondary gatekeeping in a shared media space. New Media \& Society, 16, 55-73.

Slater, M.D. (2007). Reinforcing Spirals: The Mutual Influence of Media Selectivity and Media Effects and Their Impact on Individual Behavior and Social Identity. Communication Theory, 17, 281-303.

Stier, S., Bleier, A., Lietz, H., \& Strohmaier, M. (2018). Election Campaigning on Social Media: Politicians, Audiences, and the Mediation of Political Communication on Facebook and. Twitter. Political Communication, 35, 50-74.

Suhay, E., Bello-Pardo, E., \& Maurer, B. (2017). The Polarizing Effects of Online Partisan Criticism: Evidence from Two Experiments. The International Journal of Press/Politics, 23, 95-115.

Tan, Y., \& Weaver, D. H. (2013). Agenda Diversity and Agenda Setting From 1956to 2004: What are the trends over time? Journalism Studies, 14, 773-789.

Tromble, R. (2016). Thanks for (actually) responding! How citizen demand shapes politicians' interactive practices on Twitter. New Media \& Society, 20, 676-697.

Tung, M. (2019). diffbot-r-client. https://github.com/diffbot/diffbot-r-client. Zugegriffen: 25. Okt. 2019.

Van Cuilenburg, J. (1999). On Competition, Access and Diversity in Media, Old and New: Some Remarks for Communications Policy in the Information Age. New Media \& Society, 1(2), 183-207.

Voakes, P. S., Kapfer, J., Kurpius, D., \& Chern, D. S.-Y. (1996). Diversity in the News: A Conceptual and Methodological Framework. Journalism \& Mass Communication Quarterly, 73, 582-593.

Wallace, J. (2018). Modelling Contemporary Gatekeeping: The rise of individuals, algorithms and platforms in digital news dissemination. Digital Journalism, 6, 274-293.

Dr. Gerret von Nordheim ist PostDoc im Fachgebiet Journalistik und Kommunikationswissenschaft der Universität Hamburg

Jonas Rieger ist wissenschaftlicher Mitarbeiter an der Fakultät Statistik der TU Dortmund. 